\title{
An Interferometric Study of CO and CS in Comet Hale-Bopp (C/1995 O1)
}

\author{
Y.-J. Kuan ${ }^{1,2}$, M.-L. Pen ${ }^{1}$, H.-C. Huang ${ }^{1}$, J. M. Veal ${ }^{3}$ \& L. M. \\ Woodney $^{4}$ \\ 1 National Taiwan Normal University, Taipei, Taiwan, ROC \\ 2 Academia Sinica Institute of Astronomy $\&$ Astrophysics, Taiwan \\ 3 Southwestern College, CA 91910, USA \\ 4 Lowell Observatory, Flagstaff, AZ 86001, USA
}

\begin{abstract}
We used the BIMA Array to observe CO and CS molecules in Comet Hale-Bopp (C/1995 O1) during the 1997 apparition. A spurlike structure around the cometary nucleus is observed. These molecular spurs may be attributed to gas or dust jets. Using Haser model, we find the theoretically predicted $\mathrm{CO}$ scale length agrees well with observations. A large fraction of $\mathrm{CO}$ is sublimated directly from the nucleus, with the remaining $\mathrm{CO}$ coming from icy dust grains in cometary coma. The $\left[\mathrm{CO} / \mathrm{H}_{2} \mathrm{O}\right]$ abundance ratio of Hale-Bopp is consistent with that of icy dust grains in star forming regions. We conclude that Hale-Bopp was likely formed at the stage when there were still ample of icy grains in Solar Nebula; therefore, abundances of parent molecules in a comet may reflect the primordial chemical composition of the Nebula.
\end{abstract}

\section{Introduction}

Comets are thought to have their chemical compositions preserved from pristine interstellar material in Solar Nebula. However, individual comets are known to be different from one another sometimes. By comparing cometary chemical abundances with interstellar medium in star-forming regions, we will better understand the origin of comets, the physical conditions and chemical compositions of the primordial Nebula, and the formation of our solar system accordingly. In addition, study of cometary compositions will also provide us important clues to the physical and chemical evolution of parent molecular clouds, which form planetary systems eventually. The contemporary view is that much of the organic material required to initiate the Earth's prebiotic chemistry was delivered by cometary and asteroidal impacts (e.g., Chyba et al. 1990). Perhaps this is one of the most profound reasons to study comet for its possible connection with the origin of life in our solar system; nevertheless, such a relation between comets and life is yet to be proven by direct observational evidence.

\section{Observations and Results}

Comet Hale-Bopp was observed at $115.27 \mathrm{GHz}(\mathrm{CO} \mathrm{J}=1-0)$ and at $97.98 \mathrm{GHz}$ (CS J=2-1) using the BIMA (Berkeley-Illinois-Maryland Association) Array 

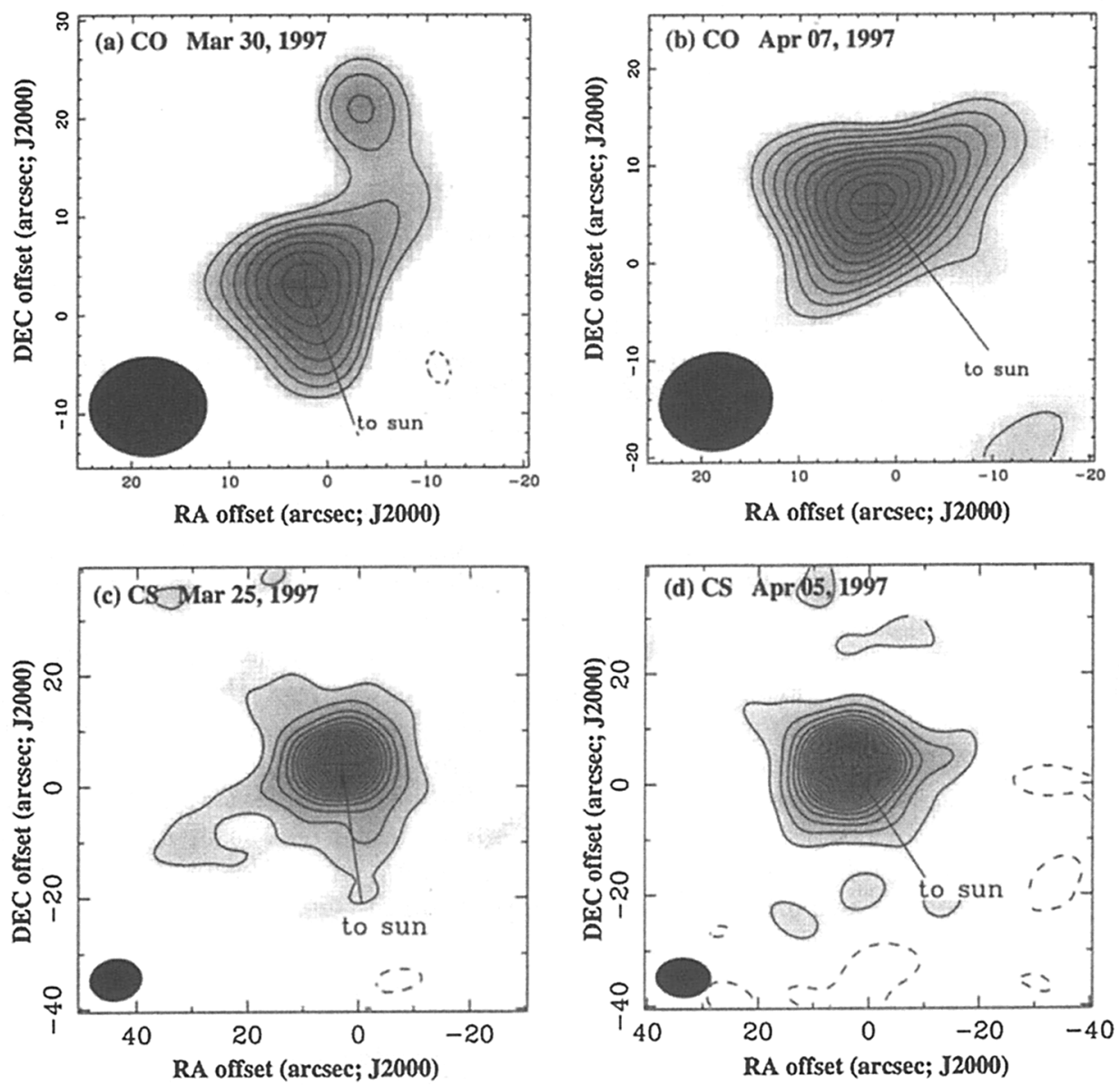

Figure 1. The integrated-intensity maps of $\mathrm{CO}$ and CS

from March 25 to April 7 during the 1997 apparition. Figure 1 shows the integrated intensity maps of CO [Figs. 1(a) \& (b)] and CS (Figs. 1(c) \& (d)) of Hale-Bopp. The gas production rate, $Q$, is a measure of how many molecules of certain species are leaving the cometary surface per second. The typical gas production rate (at $1 \mathrm{AU}$ ) of water is $Q \simeq 10^{30} s^{-1}$, for example. Considering photodissociation when molecules are exposed to solar UV radiation, the Haser model attempts to derive the column densities, $N$ in $\mathrm{cm}^{-2}$, of the parent and daughter molecules of a comet. Haser model is particularly useful for an icy halo surrounding the comet nucleus, such as in the case that parent molecular emission extends well beyond the photodissociation scale length $\left(\Lambda_{p}\right)$ when parent molecules are sublimated from both the comet nucleus and surfaces of dust grains in the halo. Assuming a spherical cometary nucleus releasing parent molecules at a constant outflow velocity, $v$, and the photodissociation rate is directly proportional to the number of parent molecules, we have the Haser 
model for parent molecules:

$$
N_{p}(a)=\frac{Q}{2 \pi v} e^{r_{n} / \Lambda_{p}} \int_{0}^{\infty} \frac{e^{-\sqrt{a^{2}+z^{2}} / \Lambda_{p}}}{a^{2}+z^{2}} d_{z}
$$

where $r_{n}$ is the radius of the nucleus, $z$ is the linear scale along the line of sight and $a$ is the impact parameter. Comparisons of Haser model images with the actual molecular emission observed (Fig. 1) allow us to test whether $\mathrm{CO}$ is a parent molecule or not. Figure 2 shows the Haser-model subtracted $\mathrm{CO}$ difference maps of Hale-Bopp. Little is left in the residual maps after model-image subtraction except the northwestern component seen in the image on March 30 (Fig. 2(a)) and a couple of $\$ 3-\sigma$ components of excess-emission. The northwestern component is in the direction of the dust tail which indicates a minority of $\mathrm{CO}$ gas toward the comet might be sublimated from icy dust grains in the comet halo; alternatively, though less likely, these $\mathrm{CO}$ molecules may be in fact daughter molecules produced via photodissociation of other parent molecules. The overall agreement between the observed $\mathrm{CO}$ emission and Haser model strongly suggests $\mathrm{CO}$ is a parent molecule coming primarily from the comet nucleus. Cometary CO abundance, therefore, may well reflect the primordial abundance of the primitive Solar system bodies. The theoretically predicted photodissociation scale length of $\mathrm{CO}$ (Huebner et al. 1992) is also confirmed by the rather clean difference maps. The observed column density at $1 \mathrm{AU}$ is $2.31 \times 10^{16}$ $\mathrm{cm}^{-2}$ for $\mathrm{CO}$, and $2.32 \times 10^{14} \mathrm{~cm}^{-2}$ for CS; the gas production rates thus derived are $Q=3.56 \times 10^{30} \mathrm{~s}^{-1}$ and $4.03 \times 10^{28} \mathrm{~s}^{-1}$ of CO and CS, respectively. A $\left[\mathrm{CO} / \mathrm{H}_{2} \mathrm{O}\right]$ abundance ratio of $\sim 0.36$ is obtained, based on the $\mathrm{H}_{2} \mathrm{O}$ production rate measured by Dello Russo et al. (2000); and for $\left[\mathrm{CS} / \mathrm{H}_{2} \mathrm{O}\right], \sim 0.004$.
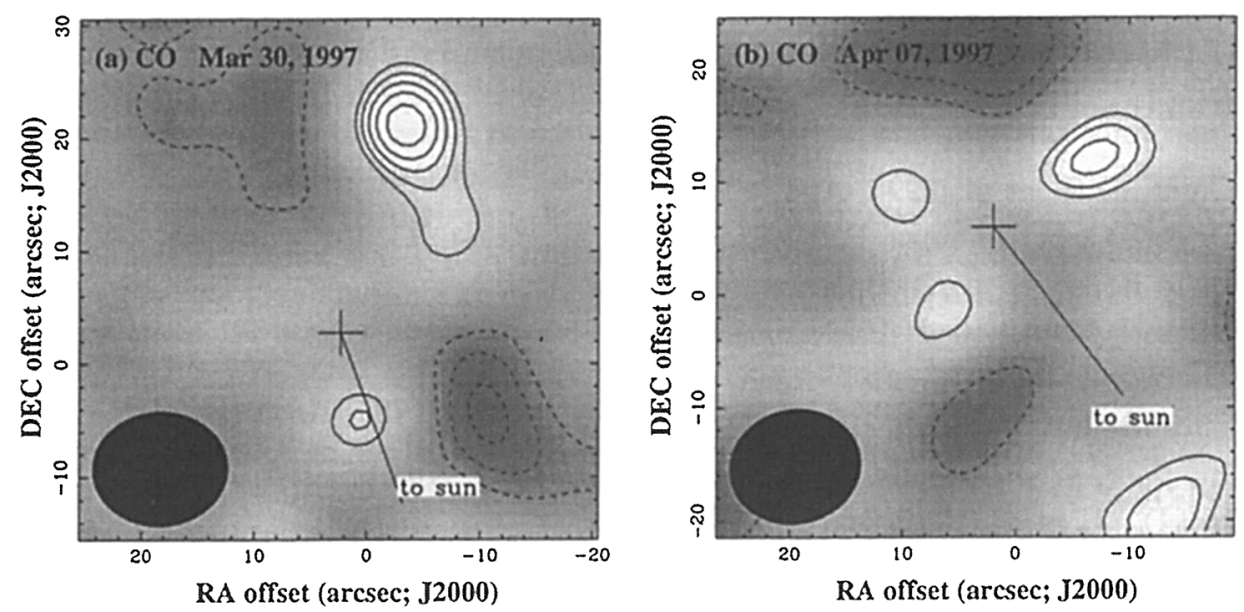

Figure 2. Haser-model subtracted $\mathrm{CO}$ difference maps. The excess emission (the positive residuals shown in solid contours) is attributed to $\mathrm{CO}$ sublimated from surfaces of icy dust grains in the comet halo. 


\section{Discussions}

The $\left[\mathrm{CO} / \mathrm{H}_{2} \mathrm{O}\right]$ abundance ratio derived is consistent with that measured by DiSanti et al. $(1999 ; \sim 0.24)$. When compared to other comets, the abundance ratio of Hale-Bopp (Period $₹ 3000$ years) is similar to that of other long-period comets ( $\mathrm{P}>200$ years), such as $\sim 0.43$ (Womack et al. 1997) and $\sim 0.3$ (Lis et al. 1997) of Comet Hyakutake ( $\mathrm{P} \sim 20,000$ years); and is a factor of 2 higher than that of Comet Halley ( $\mathrm{P}=76$ years; Eberhardt et al. 1987). However, the $\left[\mathrm{CO} / \mathrm{H}_{2} \mathrm{O}\right]$ ratio is significantly higher than that of short-period comets $(\mathrm{P}<$ 200 years, excluding Halley-family comets). For example, Comet 21P/GiacobiniZinner $(\mathrm{P}=6.61$ years) has a ratio of only $\sim 0.02-0.03$ (Weaver et al. 1999), and Comet 103P/Hartley $2(\mathrm{P}=6.3$ years), $\leq 0.01$ (Weaver et al. 1994). Both Comet G-Z and 103P/Hartley 2 are of Jupiter-family comets. The large difference of $\left[\mathrm{CO} / \mathrm{H}_{2} \mathrm{O}\right]$ ratios between long-period and short-period comets may be largely due to the fact that long-period comets are from Oort cloud but short-period ones were formed in Kuiper Belt. However, various chemical evolution experienced by individual comets may also play an important role on the difference found.

When compared to interstellar medium (ISM), the $\left[\mathrm{CO} / \mathrm{H}_{2} \mathrm{O}\right]$ ratio of HaleBopp is comparable to that of ISM measured in solid-phase via IR observations. An abundance ratio of $\sim 0.18$ was measured towrad high-mass YSO, RAFGL 7009S (Ehrenfreund et al. 1997); likewise, 0.12 in NGC 7538 IRS 9 (Whittet et al. 1996). A ratio of 0.30 was reported in the low-mass YSO, R CrA IRS1 (Chiar et al. 1998). Nonetheless, the abundance ratios observed in gas-phase in hot molecular cores, $\sim 30$ in Orion hot core (Knacke \& Larson 1991) and $~ 4000$ in Sgr B2(N) (Sutton et al. 1991), are significantly higher. It suggests that the $\left[\mathrm{CO} / \mathrm{H}_{2} \mathrm{O}\right]$ ratios of Hale-Bopp and interstellar icy dust grains are quite similar. This strongly indicates that some comets, at least for the long-period ones, were formed when a large quantity of icy dust grains from the parent cloud were still preserved in the Nebula.

\section{References}

Chiar, J. E., et al. 1998, ApJ, 498, 716

Chyba, C. F., Thomas, P. J., Brookshaw, L., Sagan, C. 1990, Science, 249, 366

Dello Russo, et al. 2000, Icarus, 143, 324

DiSanti, M. A., et al. 1999, Nature, 399, 662

Eberhardt, P., et al. 1987, A\&A, 187, 481

Ehrenfreund, P., et al. 1997, Icarus, 130, 1

Huebner, W. F., Keady, J. J., \& Lyon, S. P. 1992, Ap\&SS, 195, 1

Lis, D. C., et al. 1997, Icarus, 130, 355

Knacke, R. F. \& Larson, H. P. 1991, ApJ, 367, 162

Sutton, E. C., et al. 1991, ApJS, 77, 255

Weaver, H. A., et al. 1994, ApJ, 422, 374

Weaver, H. A., et al. 1999, Icarus, 142, 482

Whittet, D. C. B., et al. 1996, A\&A, 315, L357

Womack, M., Festou, M. C., \& Stern, S. A. 1997, AJ, 114, 2789 